\title{
Atmospheric Pressure Plasmas in Resonant Circuits
}

\author{
S.D. Anghel ${ }^{*}, 1$, T. Frentiu ${ }^{2}$ and A. Simon ${ }^{1}$ \\ ${ }^{1}$ Faculty of Physics, Babes-Bolyai University, M. Kogalniceanu 1, Cluj-Napoca, 400084, Romania \\ ${ }^{2}$ Faculty of Chemistry, Babes-Bolyai University, Arany Janos 11, Cluj-Napoca, 400028, Romania
}

\begin{abstract}
This paper attempts to give a short overview of atmospheric pressure plasmas generated in very non-uniform radiofrequency electric fields. All these forms of gas discharges have a common characteristic: they are generated based on the possibility to obtain very high voltages in resonant series RLC circuits. The paper presents both the radiofrequency generators designed in this aim and some applications of generated plasmas, especially as spectral source for analyzing of liquid and solid samples and as bacterial deactivation agent. Thus, this paper is meant for both laboratory plasma generators designers and plasma spectroscopists.
\end{abstract}

Keywords: Plasma, resonant circuit, radio-frequency, parameters, atomic emission spectroscopy.

\section{INTRODUCTION}

Over the past decades, the interest in atmospheric pressure plasmas (APP) has increased due to their numerous applications: spectral sources for atomic emission and fluorescence spectroscopy, gas treatments and material processing (deposition of thin films, etching, ion implantation, surface activation of polymers, plasma polymerization, ashing, oxidation, surface hardening), ozone generation, environmental and biomedical applications and particle sources. Most of the aspects regarding generation, characteristics and applications of APPS were largely presented in references [1-4].

APPs overcome the disadvantages of vacuum operation but present difficulty of its sustaining because of the higher electric voltages needed for gas breakdown. In addition, it must be passed the difficulties imposed by the necessity of adapting the output impedance of the waveform generator to the plasma impedance. Commercial plasma generators solve these problems by interconnecting a matching network between the waveform generator and the plasma torch. The idea of our group was the solving of the both problems by generating the plasma as intrinsic part of a RLC series resonant circuit. The behavior of the resonant circuit represents the developing basis of some APPs generators. Plasmas with powers ranging from some hundred of $\mathrm{mW}$ to some $\mathrm{kW}$ were generated at frequencies ranging from some hundred of $\mathrm{kHz}$ to $27.12 \mathrm{MHz}$. In this paper we present a short overview on APPs generators designed and built according to this generation principle and on their applications.

\section{SERIES RESONANT CIRCUIT}

Consider a simple series circuit (Fig. 1) consisting of a coil with the inductance $L$ and the loss resistance $r$, and a capacitor with the capacitance $C$, supplied by a voltage source, $u(t)=U \sin 2 \pi f t$, with the adjustable frequency, $f$. It is

*Address correspondence to this author at the Faculty of Physics, BabesBolyai University, M. Kogalniceanu 1, Cluj-Napoca, 400084, Romania; E-mail: anghels@phys.ubbcluj.ro well known that such a circuit has a resonant behavior. The ratio between the amplitude of the voltage across the capacitor $\mathrm{C}, U_{\mathrm{C}}$, and the amplitude of the supply voltage $U$ can be expressed as:

$$
k=U_{C} / U=1 / \sqrt{\left(1-m^{2}\right)^{2}+\frac{r^{2}}{4 \pi^{2} f_{o}^{2} L^{2}} m^{2}}
$$

where, $f_{o}=1 / 2 \pi \sqrt{L C}$ and $m=f / f_{o}$.

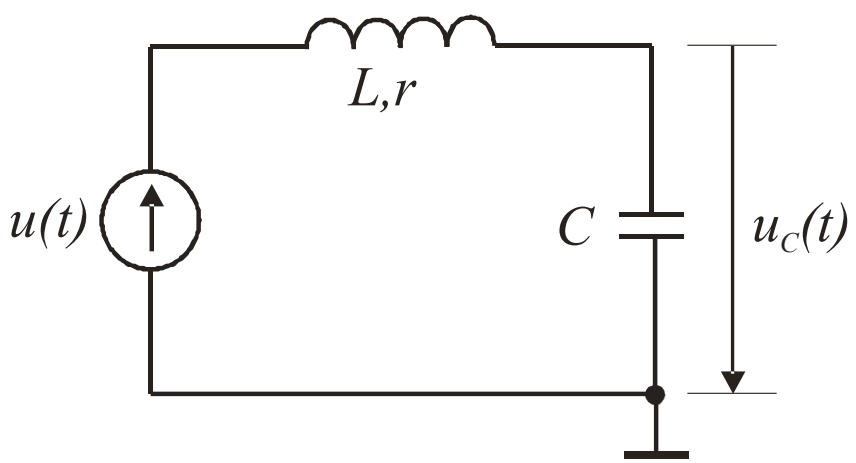

Fig. (1). RLC series circuit.

It must be mentioned that $k$ also represents the ratio between the amplitude of the voltage across the reactive part of the coil and the amplitude of the supply voltage. Having a coil with known parameters (ex. $L=12.5 \mu \mathrm{H}$, and $r=9.2 \Omega$ at $f=27.12 \mathrm{MHz}$ ), the resonant frequency will depend on the capacitance $C$. Because of the Skin effect, the loss resistance of the coil depends on the working frequency. Fig. (2) presents the dependence of the $k$ factor on the normalized frequency $m$ for three different values of $C$ and, for three different resonant frequencies, consequently.

Two important characteristics must be observed: (a) the high selective behavior at high frequencies; and (b) the possibility of obtaining across the capacitor of voltages much higher than the supply voltage at the resonant frequency $f_{\mathrm{o}}$ $(\mathrm{m}=1)$. The multiplying factor $\mathrm{k}$ at the resonant frequency also represents the quality factor of the circuit, $\mathrm{Q}$. 


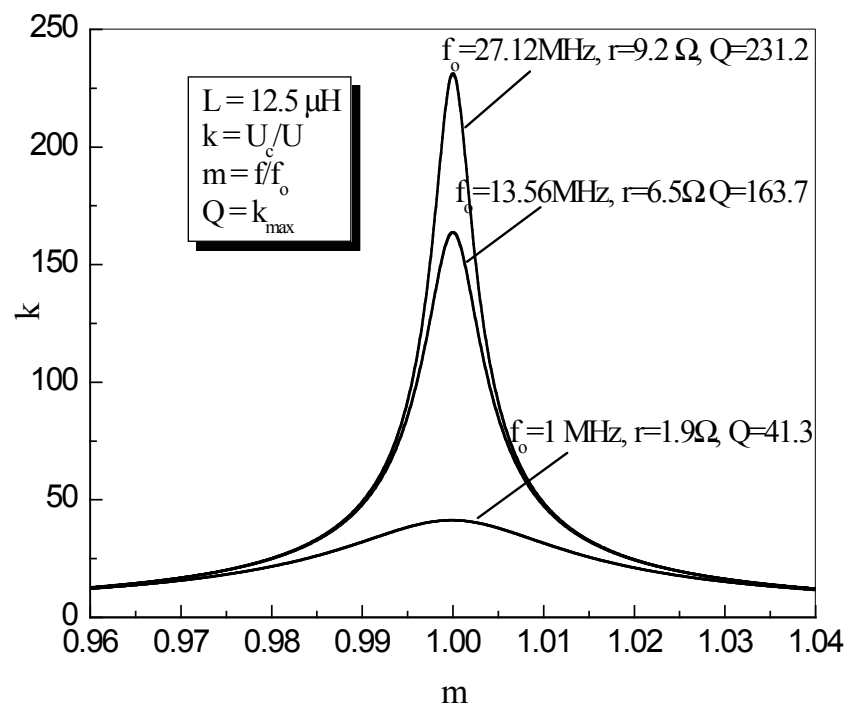

Fig. (2). Dependence of the $m$ factor on the normalized frequency for the same coil placed in three different series circuits.

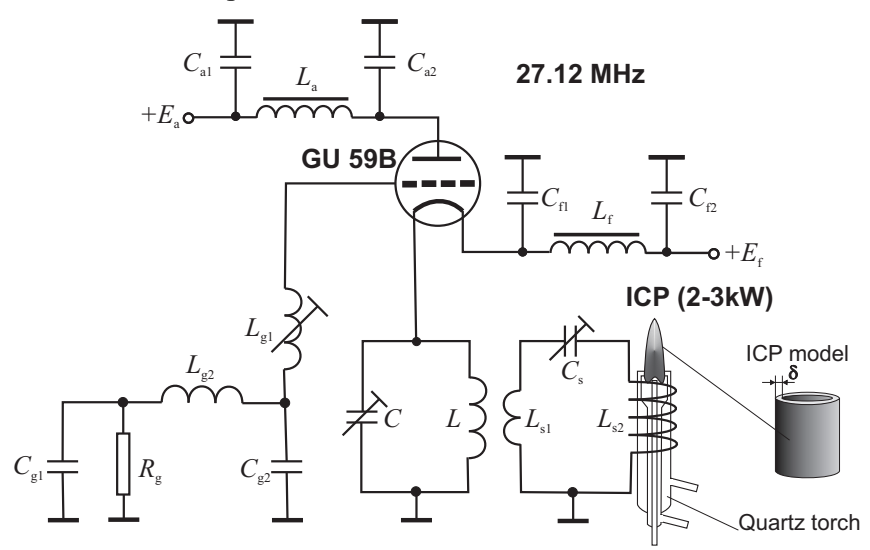

Fig. (3). Generation of ICP using inductively coupled resonant circuits.

The fact that under the resonant condition the voltages across the both reactive circuit elements of the series RLC circuit are Q times higher that the supply voltage was used by us to generate the sufficiently high voltages for generating two kinds of APPs: inductively coupled plasma (ICP) and capacitively coupled plasma (CCP). This classification is based on the physical mechanisms by which the plasma absorbs the power from the electromagnetic field. The ICP absorbs almost all its energy from the electromagnetic field via the electromagnetic induction phenomena, like a voltage transformer. The CCP absorbs its energy from the electric component of the electromagnetic field, like a real capacitor. In both cases the plasma acts as an intrinsic part of a resonant circuit.

\section{INDUCTIVELY COUPLED PLASMA AND HIGH POWER CAPACITIVELY COUPLED PLASMA}

The opportunity of using of the resonant behavior of a RLC series circuit for plasma generation was employed for the first time by Boumans [5]. He demonstrated the possibility of stabilization of the plasma power when it is generated inside the coil of the resonant circuit of a radiofrequency (rf) oscillator. The first plasma generator built by our group was one for ICP generation [6]. The simplified electric diagram of the rf oscillator is shown in Fig. (3). It is able to transfer to the plasma a power of $2-3 \mathrm{~kW}$ at a frequency of $27.12 \mathrm{MHz}$. An argon ICP is generated inside a quartz tube passing through a coil which is a component part of a resonant circuit. The resonant circuit in which the plasma is generated is inductively coupled with the resonant circuit of the free running rf oscillator. The plasma itself acts as secondary coil of the transformer consisting of the coil $L_{\mathrm{s} 2}$ and the plasma, so that the energy is transmitted from the $\mathrm{rf}$ generator to the plasma via two magnetic coupling, as can be seen in Fig. (4).

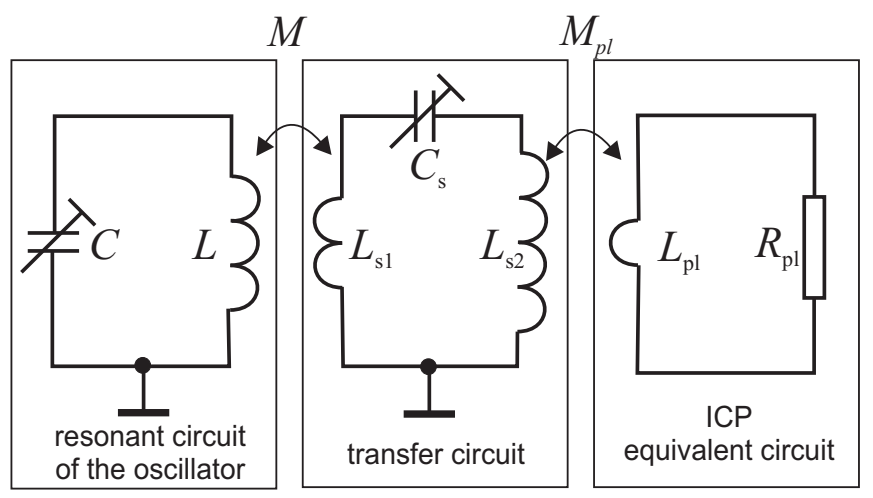

Fig. (4). Transmission of the energy to the ICP by magnetic coupling.

Three main conditions must be satisfied in order to be possible the ICP generation: (a) the voltage across the coil $L_{2 \mathrm{~s}}$ must be sufficiently high (a few kV's) so that the accelerated electrons give rise the ionization collisions, creating ions and electrons; (b) the current flowing through the transfer circuit must be very intense in order to assure a high plasma power; and (c) the flowing of the plasma gas (particularly argon) must be a helical one for contributing at the formation of the almost closed current loops. The first two conditions are realized by choosing $L_{\mathrm{s} 2} \gg L_{\mathrm{s} 1}$ and by designing a resonant circuit having a very high quality factor, $Q$. The third condition is realized by a proper construction of the quartz torch. The plasma acts as a coil with only one turn having the inductance $L_{\mathrm{p}}$ and the loss resistance $R_{\mathrm{p}}$. With good approximation, the plasma can be modeled as a metallic tube as it is shown in Fig. (3), where $\delta$ represents the Skin depth, because at so high frequencies the Skin effect can not be neglected. For example, in an ICP with a temperature of $10^{4} \mathrm{~K}$, its resistivity is about $8 \cdot 10^{-5} \Omega \cdot \mathrm{m}$ and the Skin depth is lower than $1 \mathrm{~mm}$. The presence of the plasma inside the resonant circuit which is inductively coupled with the resonant circuit of the rf oscillator gives rise to an increase in the oscillating frequency with maximum $70 \mathrm{kHz}$.

Another rf oscillator built by our group for its using as plasma generator, is one of Colpitts type (Fig. 5) [7]. It is very simple to build, has a good working stability, ensures a high transfer efficiency of the power from generator to the plasma and has smaller dimensions than the previous one. The capacitors $C_{1}, C_{2}$ and $C_{\mathrm{g} 1}$ have air as dielectric and are adjustable, making possible the generation of high voltages across the coil $L$ and a good matching of the oscillator output impedance to the plasma impedance. Due to its flexibility it can be use both for ICP or CCP generation.

Because ICP was largely studied by many researchers, in this paper does not are presented our results regarding its use 


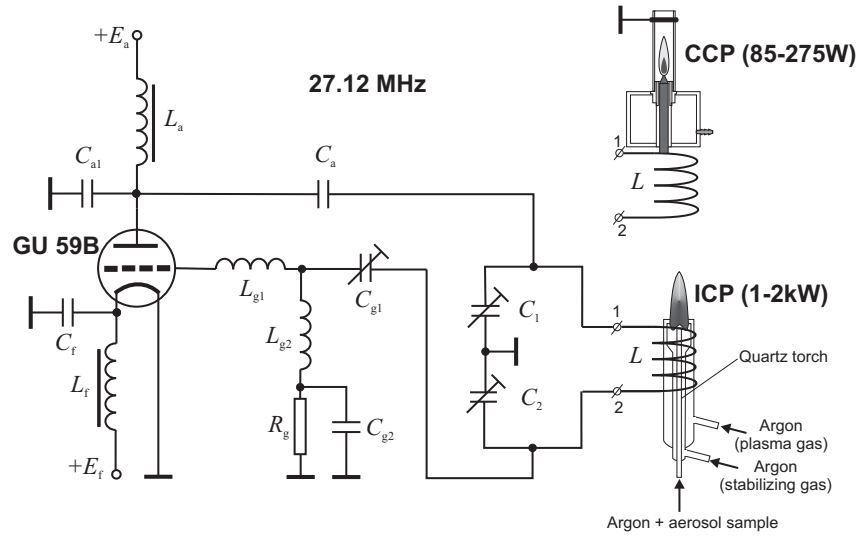

Fig. (5). Colpitts oscillator for generating ICP and high power CCP.

as spectral source. They are in accordance with the others. For about twenty years our attention is focused on the other kind of rf discharge, capacitively coupled plasma. The majority of our studies were oriented on estimation of plasma parameters and on determination of its capabilities to be used as spectral source for analyzing of liquid and solid samples by atomic emission spectroscopy (AES). Two kind of torches were build in this aim: (a) one in which the liquid sample, after its pneumatic nebulization, is introduced into the plasma, through twelve holes placed on the circle around the rf powered electrode [8], and (b) the other one, in which the rf powered electrode is tubular and the sample is introduced into the plasma through its channel [9]. The first torch was also used for analyzing of solid conductive samples [10]. The two torches are schematic shown in Figs. $(6,7)$.

The first one, which was called tip-ring torch, has one ring as grounded electrode and the latter one, called tubering torch, has one or two grounded ring electrodes. They surround the quartz tube in which the plasma is generated and contribute to finding the best spatial distribution of the electric field, so that the analytical performances are maximized.

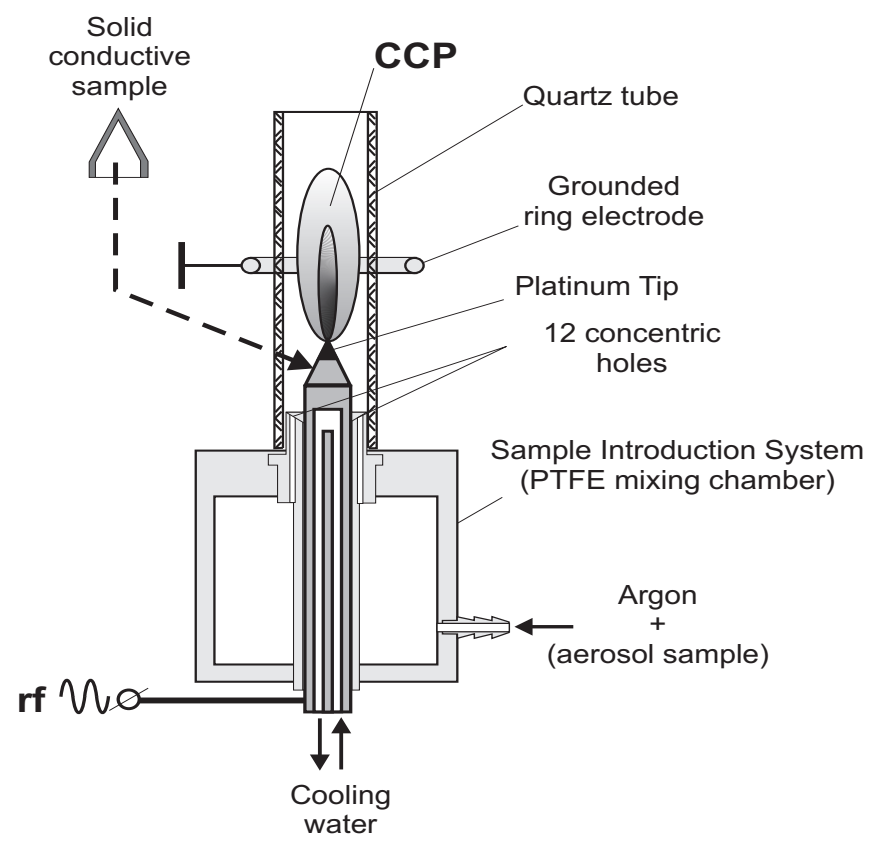

Fig. (6). Tip-ring torch for analyzing of liquid and solid conductive samples.

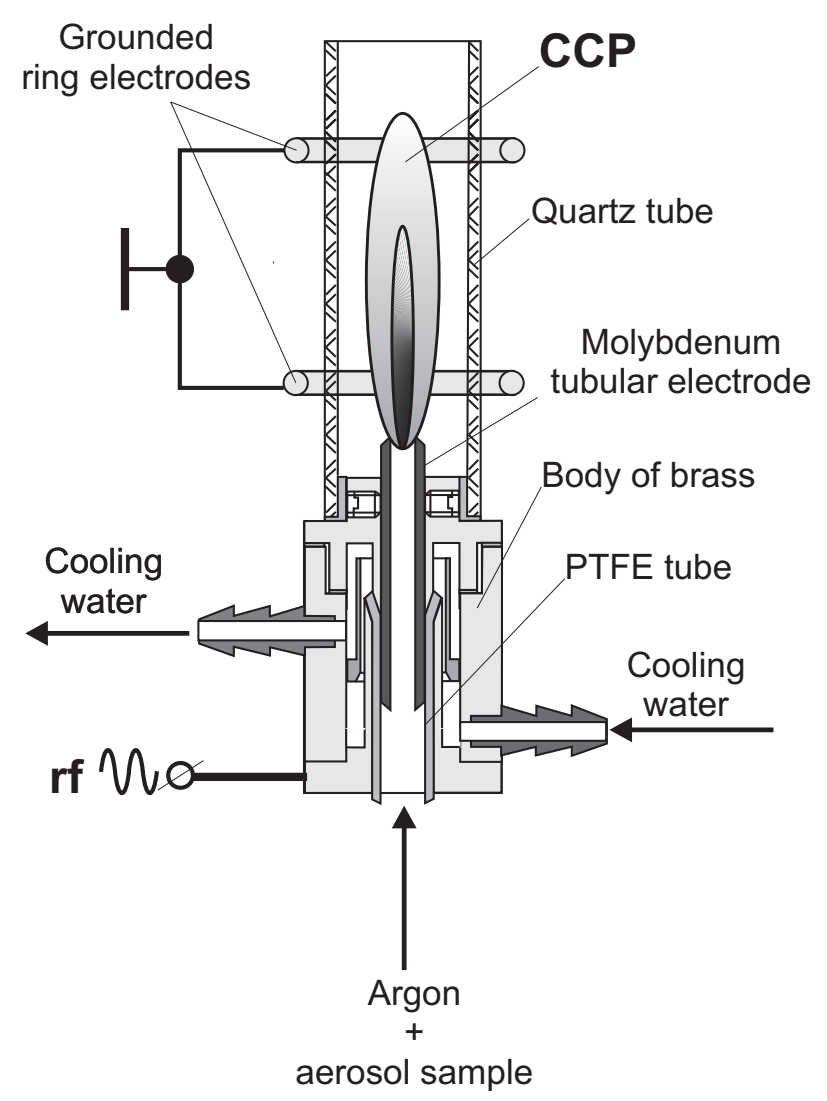

Fig. (7). Tube-ring(s) torch for analyzing of liquid samples.

In order to evaluate the physical characteristics of the plasma generated in the tip-ring electrodes geometry and its analytical capabilities in analyzing of liquid samples, the plasma radiation was collected in two ways: the radial viewing direction (perpendicular to the plasma axis) and the axial viewing direction (along the plasma axis). The samples were introduced into the plasma by pneumatic nebulization. The plasma gas (Ar with a flow-rate lower than $11 \mathrm{~min}^{-1}$ ) was also used as nebulizing gas. The limits of detection (LODs) for the studied elements and the optimum working parameters for each element are presented in Table 1. In case that the emitted radiation is collected in radial viewing mode, the observation high represents the distance from the electrode tip to the observed region of the plasma. The detailed results can be found in references [8, 10-13].

For many elements, the LODs obtained in the axial viewing mode are comparable or better than those obtained in the radial viewing mode. This is probably due to the fact that in the axial viewing mode the emitted radiation is collected from a bigger volume of the plasma and the self-absorption is negligible.

The values of the physical parameters of the plasma determined by optics-spectral methods in the axial viewing mode are approximately equals to their mean values determined in radial viewing mode at different observation heights. In the radial viewing mode, the excitation temperatures for Ar were in the range of $1800-3700 \mathrm{~K}$ and for Fe between $3700-$ $4700 \mathrm{~K}$, the ionization temperature for $\mathrm{Ca}$ was between 4400 and $4650 \mathrm{~K}$, the rotational temperature of $\mathrm{OH}$ was in the range of $1850-2200 \mathrm{~K}$ and the electron number density was estimated to be in the range of $3-510^{14} \mathrm{~cm}^{-3}$. 
Table 1. Comparation Between LODs in CCP with Tip-Ring Electrodes Geometry in Radial and Axial Viewing Modes

\begin{tabular}{|c|c|c|c|c|c|c|c|c|}
\hline \multirow[b]{2}{*}{ Element } & \multirow[b]{2}{*}{$\underset{\mathbf{n m}}{\lambda}$} & \multirow[b]{2}{*}{$\begin{array}{l}\mathbf{E}_{\text {exc }} \\
\text { eV }\end{array}$} & \multicolumn{4}{|c|}{ Radial Viewing Mode } & \multicolumn{2}{|c|}{ Axial Viewing Mode } \\
\hline & & & $\begin{array}{l}\text { Plasma } \\
\text { Power } \\
\text { W }\end{array}$ & $\begin{array}{c}\text { Electrode-Ring } \\
\text { Distance } \\
\text { mm }\end{array}$ & $\begin{array}{c}\text { Optimum } \\
\text { Observation } \\
\text { Height } \\
\text { mm }\end{array}$ & $\underset{\mathbf{n g ~ m l}^{-1}}{\text { LOD }}$ & $\begin{array}{l}\text { Plasma } \\
\text { Power } \\
\text { W }\end{array}$ & $\underset{\text { ng ml }^{-1}}{\text { LOD }}$ \\
\hline $\mathrm{Li}$ & 670.79 & 1.85 & \multirow{5}{*}{185} & \multirow{14}{*}{10} & 13 & 9 & \multirow{14}{*}{135} & 75 \\
\hline $\mathrm{Na}$ & 588.99 & 2.11 & & & 13 & 13 & & 9 \\
\hline $\mathrm{Eu}$ & 459.40 & 2.70 & & & 13 & 440 & & 600 \\
\hline $\mathrm{Ca}$ & 422.67 & 2.93 & & & 20 & 15 & & 8 \\
\hline $\mathrm{Pb}$ & 405.78 & 4.38 & & & 18 & 450 & & 680 \\
\hline $\mathrm{Fe}$ & 371.99 & 3.33 & \multirow{3}{*}{135} & & 15 & 240 & & 720 \\
\hline $\mathrm{Ni}$ & 352.45 & 3.54 & & & 15 & 1700 & & 1400 \\
\hline Co & 345.35 & 4.02 & & & 13 & 2500 & & 940 \\
\hline $\mathrm{Ag}$ & 328.07 & 3.78 & \multirow{6}{*}{185} & & 15 & 430 & & 170 \\
\hline $\mathrm{Cu}$ & 324.75 & 3.82 & & & 18 & 2500 & & - \\
\hline $\mathrm{Mg}$ & 285.21 & 4.34 & & & 15 & 9 & & 58 \\
\hline $\mathrm{Hg}$ & 253.65 & 4.88 & & & 18 & 750 & & 280 \\
\hline $\mathrm{Cd}$ & 228.81 & 5.41 & & & 18 & 770 & & 230 \\
\hline $\mathrm{Zn}$ & 213.85 & 5.80 & & & 18 & 700 & & 240 \\
\hline V & 437.92 & 3.13 & 185 & 20 & 9 & 620 & 135 & 1700 \\
\hline $\mathrm{Cr}$ & 425.43 & 2.91 & \multirow{3}{*}{185} & \multirow{3}{*}{25} & 13 & 120 & \multirow{4}{*}{135} & 680 \\
\hline $\mathrm{Mn}$ & 403.08 & 3.08 & & & 17 & 750 & & 1000 \\
\hline Mo & 386.41 & 3.20 & & & 13 & 520 & & - \\
\hline $\mathrm{Ba}$ & 493.41 & 2.51 & 135 & 40 & 11 & 5700 & & 2500 \\
\hline
\end{tabular}

The analytical performances of the $\mathrm{CCP}$ in tip-ring electrodes geometry were improved firstly by replacing the powered electrode having a platinum tip with a tubular electrode made of molybdenum and secondly by adding a supplementary grounded ring electrode [14-19]. The optimum position of the first grounded ring electrode was found to be at a distance of $5 \mathrm{~mm}$ on the exit nozzle of the tubular electrode. The presence of the second grounded electrode changes the electric field configuration and consequently the shape of the plasma. The plasma power was of $275 \mathrm{~W}$ and the plasma (and nebulizing) gas was Ar with a flow rate of $0.41 \mathrm{~min}^{-1}$. The LODs obtained with the three torches in radial viewing mode are comparatively presented in Table $\mathbf{2}$. Two main remarks can be done: (a) the LODs can be improved by replacing the tip electrode with the tubular electrode and by adding a second grounded ring electrode, and (b) the optimum observation high decreases when the plasma is generated with the tubular electrode and two grounded ring electrodes, unlike the situation when the plasma is generated with torches having only one grounded ring electrode. It must be mention here that some LODs obtained in $\mathrm{rf}$ CCP generated with the tubular-two grounded ring electrodes are better than those obtained in microwave induced plasma and comparable with those obtained in inductively coupled plasma.
The high power Ar CCP (135 W, 27.12 MHz) was also used for qualitative and quantitative analysis of low and medium alloyed steel and of brass $[10,20]$. Once the conductive sample was machining as it is shown in Fig. (6), it was placed on the powered electrode. Now, it represents one of the two electrodes of the plasma torch. Because of the electrodes asymmetry, the space closed to the sample has all the characteristics of a normal drop cathode voltage. The analyzing method is based on the sputtering of the atoms from the sample by the positive ions accelerated in this space. The sputtered atoms are transported by the argon flow into the plasma where they are excited. The emitted de-excitation radiation is analyzed by AES methods. The intensity of the emitted radiation is the highest and the relative standard deviation is the lowest when the distance between the two electrodes is of $60 \mathrm{~mm}$ and the Ar flow-rate is of $0.61 \mathrm{~min}^{-1}$. Table 3 presents the LODs of the analyzed elements and the corresponding wavelengths. The analytical results are in good agreement with the certified values.

\section{LOW AND VERY LOW POWER CAPACITIVELY COUPLED PLASMA}

For generating low and very low power atmospheric pressure CCPs, a dedicated generator was designed and built [21]. Its electric diagram is sown in Fig. (8). 
Table 2. Comparation Between LODs in CCP with Tip-Ring and Tube-Ring(s) Electrodes Geometry in Radial Viewing Mode

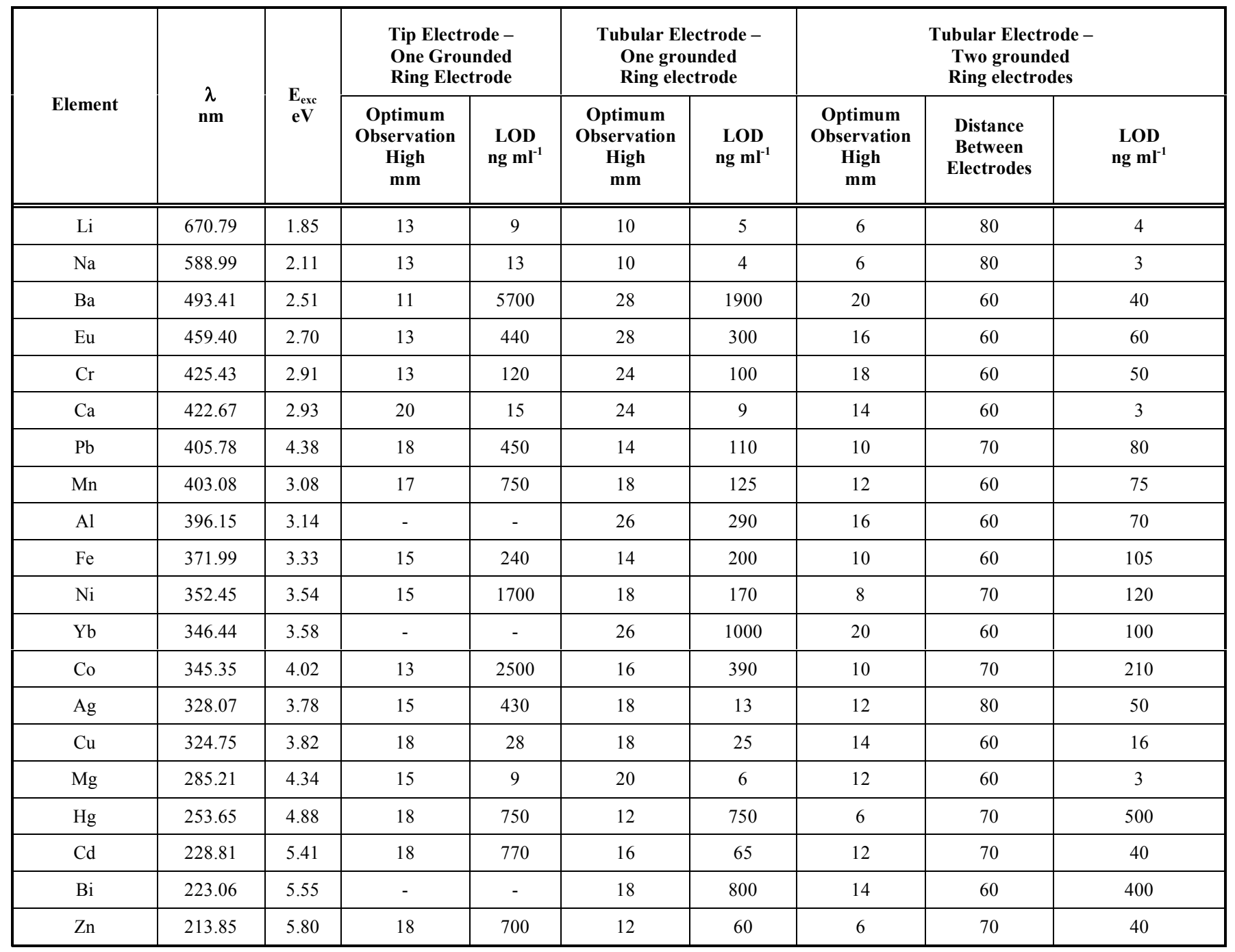

The rf oscillator is built according to a classical set-up, but having some special characteristics for the present purpose. Hence, taking into consideration the use of the plasma as spectral source, any noise produced electrically or mechanically must be eliminated. Any kind of noise can give rise to a variation of the electric parameters of the plasma, therefore a variation of the absorbed power into the plasma and, consequently, a variation of the atomization and excitation capability of the plasma. For reducing the influence of the noise on the plasma, some precautions must be taken. First, for diminishing the noise at $50 \mathrm{~Hz}$, besides supplying the anode and screen grid of the oscillating lamp (penthode) from stabilized sources of continuous voltage (dc), the supply for the filament is also a dc one. In the filament and the screen grid circuits, low pass filters $\left(\mathrm{R}_{\mathrm{f}} \mathrm{C}_{\mathrm{f}}\right.$ and $\mathrm{R}_{\mathrm{g} 2} \mathrm{C}_{\mathrm{g} 2}$, respectively) are also present. Secondly, in the mechanical execution of the generator we eliminated all kinds of instability that could give rise to plasma noise.

In all our applications the plasma is in contact with a single electrode which is in electric contact with the free end of the coil of the resonant circuit of the oscillator. Function of the specific application, a particular torch was designed and built. Four kinds of plasma torches were developed: (a) the first one for atomization and excitation of liquid samples; (b) the second one for rf sputtering and excitation of nonconductive solid samples; (c) the third one for plasma jet generation, and (d) the fourth one for very cold (nonthermal) plasma generation. Details about construction, dimensions and the used materials can be found in references [21-24].

For analyzing of liquid samples the plasma is ignited inside of a quartz tube on a kanthal ${ }^{1}$ wire fixed into the brass electrode (Fig. 9). The liquid sample is pneumatically nebulized and is introduced into the plasma by means of the device comprised of two polytetraflourethylene (PTFE) pieces, which form a chamber fixed on the brass electrode. The chamber also supports the quartz tube. From here the sample is swept into the base of the plasma trough 12 parallel channels, concentrically placed around the brass electrode and the kanthal wire. The dimensions of the chamber and the diameter of the holes, provide for a laminar flow of gas up to 1.61 $\mathrm{min}^{-1}$. When the sample is introduced into the plasma, the

${ }^{1}$ Kanthal is an alloy with high electrical resistance and high refractoriness, having the next composition: Co $2 \%$; Cr 20-24 \%; Al 5-6\% and Fe 68-73 $\%$. 
inelastic processes give rise to its dissociation, atomization, excitation and ionization.

Table 3. LODs in Conductive Solid Samples

\begin{tabular}{|c|c|c|}
\hline \multirow{2}{*}{ Element } & $\begin{array}{c}\lambda \\
\text { nm }\end{array}$ & $\begin{array}{c}\text { LOD } \\
\text { \% }\end{array}$ \\
\hline \hline \multicolumn{3}{|c|}{ Low and medium alloyed steel } \\
\hline $\mathrm{Al}$ & 396.15 & 0.003 \\
\hline $\mathrm{Co}$ & 345.35 & 0.001 \\
\hline $\mathrm{Cr}$ & 425.43 & 0.008 \\
\hline $\mathrm{Cu}$ & 324.75 & 0.006 \\
\hline $\mathrm{Mn}$ & 403.07 & 0.048 \\
\hline $\mathrm{Mo}$ & 386.41 & 0.012 \\
\hline $\mathrm{Ni}$ & 352.45 & 0.031 \\
\hline $\mathrm{V}$ & 437.92 & 0.001 \\
\hline \multicolumn{2}{|c|}{ Brass } \\
\hline $\mathrm{Pb}$ & 405.78 & 0.03 \\
\hline
\end{tabular}

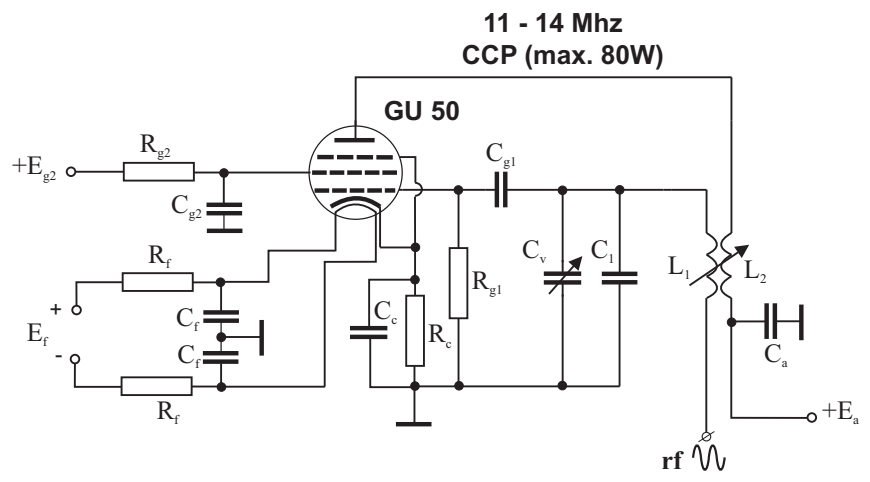

Fig. (8). Oscillator for generating low and very low power CCPs.

This kind of argon plasma (power, $60 \mathrm{~W}$, Ar flow-rate, $0.71 \mathrm{~min}^{-1}$ and observation high, $6 \mathrm{~mm}$ ) was tested as a spectral source for the analysis of pneumatically nebulized liquid samples [25]. The LODs for the studied elements are presented in Table 4. At the observation high, the rotational temperature was found to be of $2600 \mathrm{~K}$ and the excitation temperatures for $\mathrm{Ar}, \mathrm{Pb}$ and $\mathrm{Fe}$ were, respectively: $3800 \mathrm{~K}$, $3900 \mathrm{~K}$ and $5300 \mathrm{~K}$. The ionization temperature for $\mathrm{Ar}$ is around $6000 \mathrm{~K}$ and the electron number density was estimated at about $310^{13} \mathrm{~cm}^{-3}$.

Recently, the same torch was used to generate a low power capacitively coupled helium plasma (13.56 $\mathrm{MHz}$, 4-35 W) [22]. Function of working parameters, the excitation, vibrational and rotational temperatures range from 1880 to $5660 \mathrm{~K}, 1960$ to $3150 \mathrm{~K}$ and 760 to $1398 \mathrm{~K}$, respectively. The temperatures depend on the plasma power, plasma volume and gas dynamic flowing. During this first stage of our research, the atomization and excitation capability for three easily excitable elements ( $\mathrm{Zn}, \mathrm{Na}$ and $\mathrm{Li}$ ) was only tested. A systematic study over more elements will be done in the next future. The visual appearance of plasma containing $\mathrm{Na}$ and $\mathrm{Li}$ is shown in the photograph from Fig. (10a, b) shows the same image after its processing using the "posterize" tool

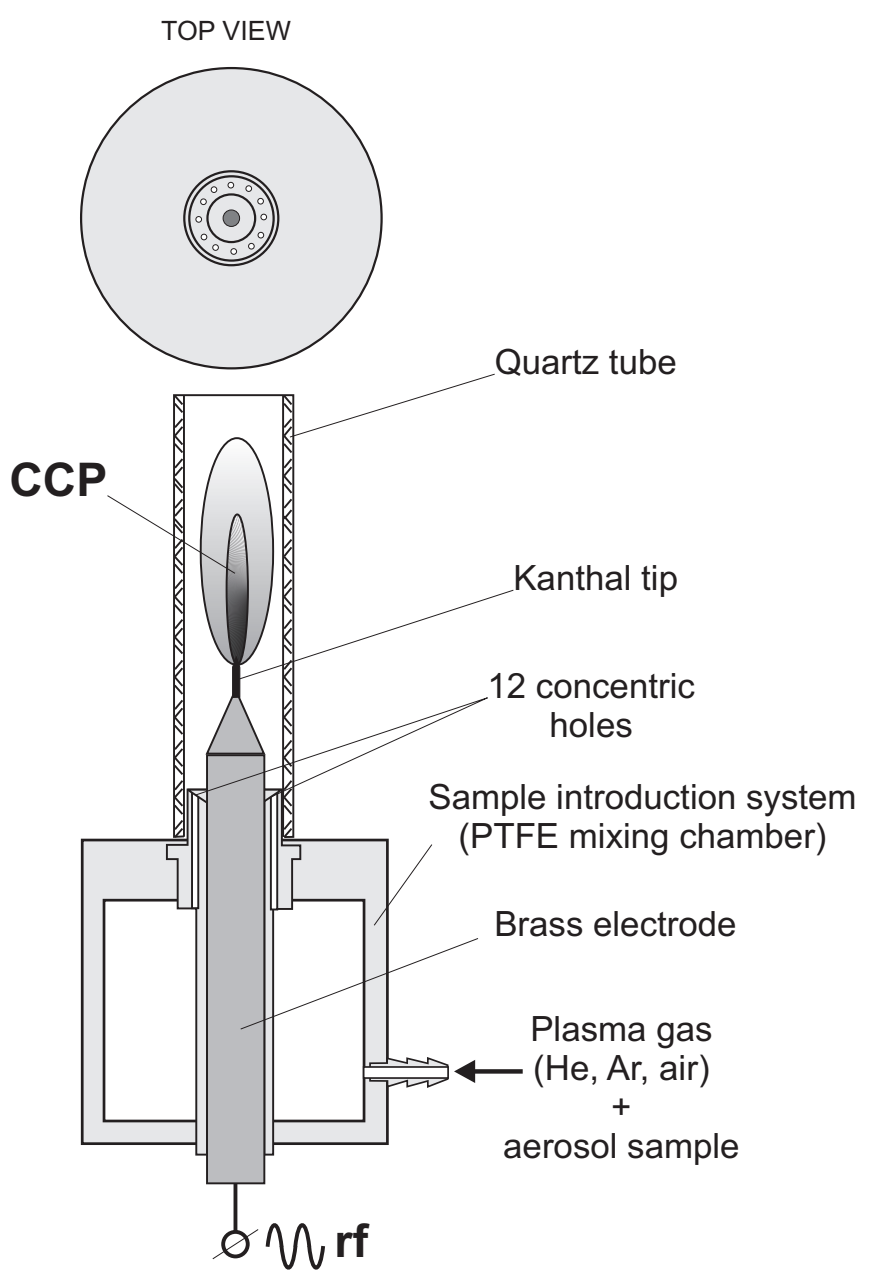

Fig. (9). Torch for analyzing of liquid samples in low power CCP.

Table 4. LODs in Low Power Ar CCP

\begin{tabular}{|c|c|c|}
\hline Element & $\underset{\mathrm{nm}}{\lambda}$ & $\underset{\mu \mathrm{g} \mathrm{ml}^{-1}}{\text { LOD }}$ \\
\hline $\mathrm{Li}$ & 670.79 & 0.13 \\
\hline $\mathrm{Na}$ & 588.99 & 0.11 \\
\hline $\mathrm{Sr}$ & 460.70 & 0.78 \\
\hline $\mathrm{Cr}$ & 425.43 & 4.20 \\
\hline $\mathrm{Ca}$ & 422.67 & 0.79 \\
\hline $\mathrm{Pb}$ & 405.78 & 1.40 \\
\hline $\mathrm{Mn}$ & 403.08 & 0.52 \\
\hline $\mathrm{Fe}$ & 373.48 & 3.75 \\
\hline $\mathrm{Ni}$ & 352.45 & 1.92 \\
\hline Co & 345.35 & 4.09 \\
\hline $\mathrm{Ag}$ & 328.07 & 0.11 \\
\hline $\mathrm{Cu}$ & 324.75 & 0.17 \\
\hline $\mathrm{Mg}$ & 285.21 & 0.10 \\
\hline $\mathrm{Hg}$ & 253.65 & 0.44 \\
\hline $\mathrm{Cd}$ & 228.81 & 0.20 \\
\hline $\mathrm{Zn}$ & 213.85 & 0.80 \\
\hline
\end{tabular}


from Microsoft Photo Editor. As it can be seen, the plasma zones in which the two elements are well excited are different. This is important in finding of the well appropriate viewing point for examination by atomic emission spectroscopy method.

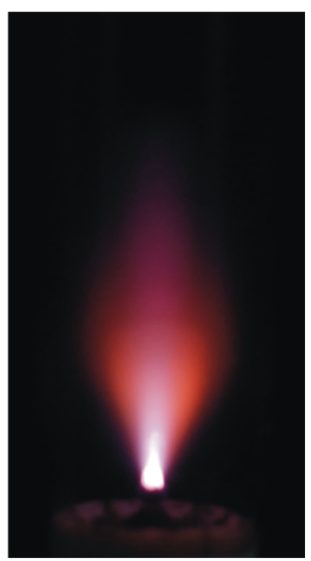

(a)

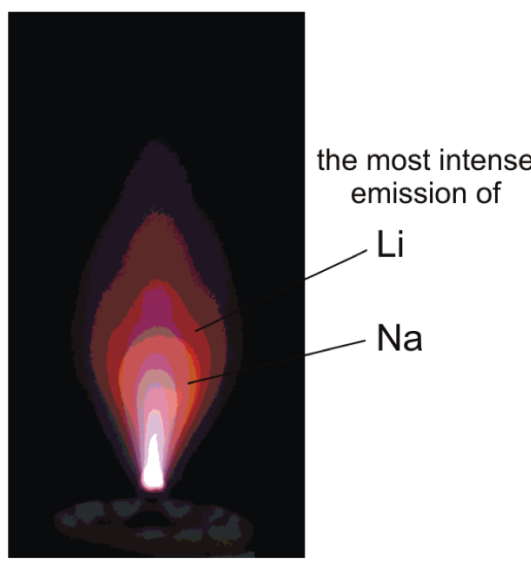

(b)
Fig. (10). (a) photograph of He plasma in which $\mathrm{Na}$ and $\mathrm{Li}$ in concentration of $200 \mu \mathrm{g} \cdot \mathrm{ml}^{-1}$ were introduced by pneumatic nebulization from liquid sample; (b) the same image after its processing using the "posterize" tool from Microsoft Photo Editor.

The torch used for rf sputtering of non-conductive solid samples was realized by remodeling the previous torch (Fig. 11). The plasma is generated on a sharp tungsten electrode inside a quartz tube provided with a lateral port. The sample pellet is placed on a plate brass counter-electrode, inside of a PTFE sample holder cup. The rf electrode and the sample holder were placed at the opposite sides of the quartz tube. The lateral port ensures the plasma viewing.

The sputtering mechanism could be explained based on the working principle of the rf oscillator. The automatic grid bias network $\left(\mathrm{R}_{\mathrm{g}} \mathrm{C}_{\mathrm{g}}\right.$, Fig. 8) develops a negative voltage on the grid. Its value is a function of the anode positive bias. The rf component of the generated waveform, having an amplitude of $15-20 \%$ greater than the dc potential, is superimposed over the negative dc voltage of the grid. Because the oscillator runs under the resonant conditions, the amplitude of the rf oscillations on the sustaining electrode of the plasma is very high $\left(\approx 10^{3} \mathrm{~V}\right)$. Due to the dc voltage component, the rf voltage component is translated toward the negative values. As a consequence, during a period of the $\mathrm{rf}$ waveform the plasma sustaining electrode has a negative voltage a time interval longer than a half-period. This fact, combined with the lower mobility of the positive ions relative to that of the electrons, determine an accumulation of positive charge greater than the negative one close to the sustaining plasma electrode. This positive charge excess has two consequences: (a) an excess of negative charge at the end of the plasma where the sample is placed, and (b) the electric shielding of the plasma electrode. Consequently, an internal dc electric field appears into the plasma. When the distance between the plasma electrode and the sample is $2-4$ $\mathrm{mm}$, this field is sufficiently intense to accelerate the positive ions towards the sample and to induce its sputtering. Sputtered atoms are then available to be diffused into the plasma for subsequent excitation and ionization. At the plasma pow- ers higher than $35 \mathrm{~W}$ the heating of the sample is so high that the local thermal vaporization takes place as a secondary mechanism of atoms generation.

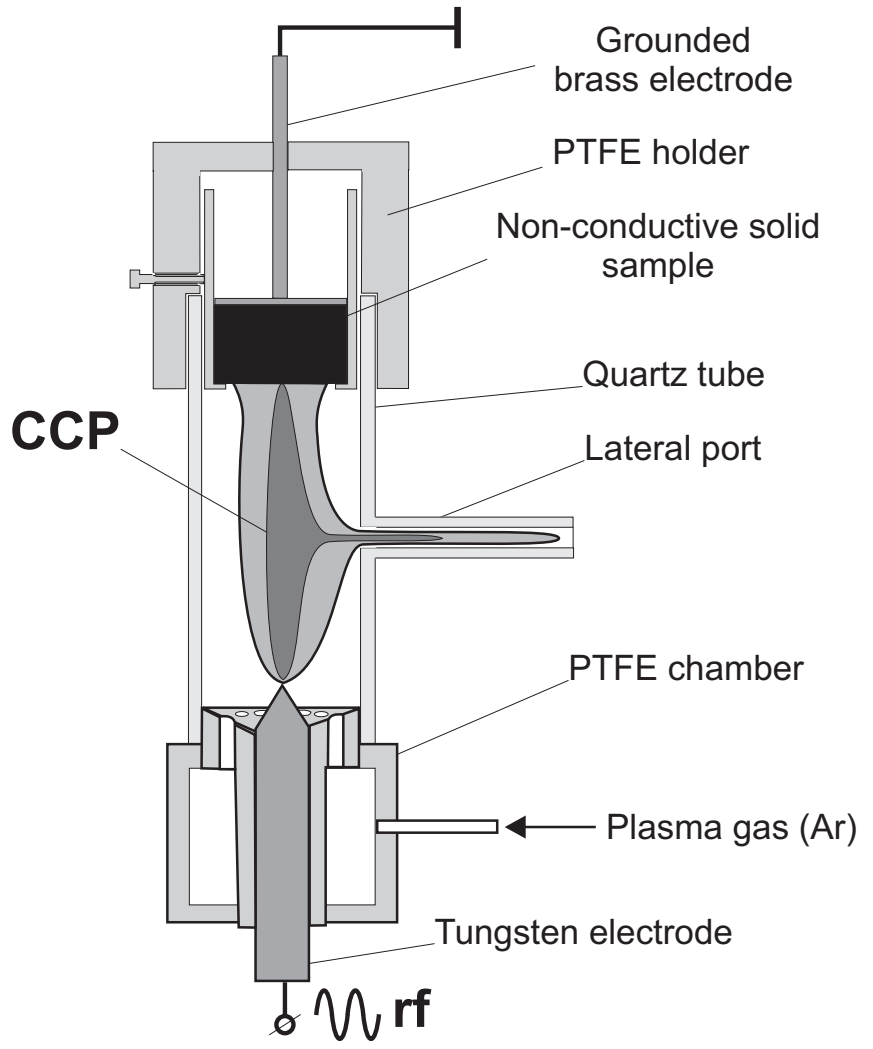

Fig. (11). Torch for sputtering and exciting of non-conductive solid samples in low power CCP.

The expulsion mechanism and the analytical performances were studied using the following materials: $\mathrm{ZnO}$ with known content of trace elements, andesite standards with certified contents of elements and synthetic (laboratory made) andesite standards [23]. The measurements for evaluating the analytical performances were made at optimum (previous determined) working parameters: plasma power, $36 \mathrm{~W}$ and argon flow rate, $0.51 \mathrm{~min}^{-1}$. The LODs for analyzed elements are shown in Table 5. They are lower than 1 $\mu \mathrm{g} \mathrm{g}^{-1}$. The results obtained for elemental concentration are in good agreement with the certified values.

Table 5. LODs for Trace Elements in $\mathrm{ZnO}$ and Andesite Matrixes

\begin{tabular}{|c|c|c|c|}
\hline Element & $\underset{\mathbf{n m}}{\lambda}$ & $\underset{\mu \mathrm{g} \cdot \mathrm{g}^{-1}}{\text { LOD }}$ & Matrix \\
\hline $\mathrm{Na}$ & 588.99 & 0.3 & \multirow{4}{*}{$\mathrm{ZnO}$} \\
\hline $\mathrm{Pb}$ & 405.78 & 0.7 & \\
\hline $\mathrm{Si}$ & 251.61 & 1.0 & \\
\hline $\mathrm{Cd}$ & 228.81 & 0.9 & \\
\hline $\mathrm{Pb}$ & 405.78 & 0.8 & \multirow{3}{*}{ Andesite } \\
\hline $\mathrm{Cr}$ & 425.43 & 1.0 & \\
\hline $\mathrm{Cu}$ & 324.75 & 0.5 & \\
\hline
\end{tabular}


The torch used for generating of plasma jet consists of a cylindrical sinterized alumina $\left(\mathrm{Al}_{2} \mathrm{O}_{3}\right)$ piece with a longitudinal channel, a glass tube and an insulating support (Fig. 12). The rf powered electrode is positioned perpendicularly to the longitudinal axis of the torch, having its tip $(1 \mathrm{~mm}$ diameter, kanthal made) centered to the exit nozzle of the alumina piece. Preliminary studies showed that by increasing the He flow-rate from 1 to $31 \mathrm{~min}^{-1}$, the jet length increases from 6 to $15 \mathrm{~mm}$ and the plasma power increases from 3 to $38 \mathrm{~W}$ [24]. The excitation, vibrational and rotational temperatures range from 1500 to $2350 \mathrm{~K}, 3500$ to $4400 \mathrm{~K}$ and 450 to $1100 \mathrm{~K}$, respectively and the electron number densities are in the range of $10^{13}-10^{14} \mathrm{~cm}^{-3}$.

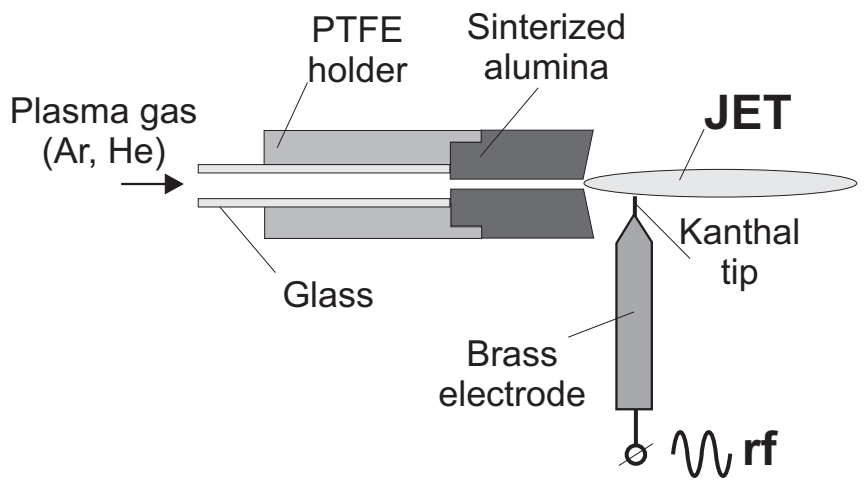

Fig. (12). Torch for generating a low power plasma jet.

Very cold and non-aggressive atmospheric pressure helium plasmas were generated at $13.56 \mathrm{MHz}$ and $0.9 \mathrm{MHz}$ using the previous described generator and a special dedicated one, respectively. The latter, of which diagram is presented in Fig. (13), was recently designed and built [26, 27]. It is a very simple and efficient very low power plasma generator. The most important of its components are the ferritecore transformer and the plasma torch. The series resonant circuit is represented by the secondary coil of a ferrite-core transformer and by the torch. It is powered by a voltage source representing the induced electromagnetic voltage from the primary coil. From a function generator, a triggering signal (square waveform with adjustable duty cycle) is applied on the MOSFET (metal-oxide-semiconductor fieldeffect transistor) gate. The transistor channel will represent a very low or a very high resistance connected between the primary coil and the ground, depending on the triggering signal voltage. As a consequence, through the primary coil will flow current pulses representing the excitation pulses for the secondary resonant circuit. The higher conversion efficiency of dc power into the plasma power is obtained when the triggering signal has the same frequency as the resonant frequency of the secondary circuit and a duty cycle of about $87 \%$. One end of the secondary coil is firmly grounded and the other one is connected to the female part of a BNC connector.

Fig. (14) shows the plasma torch used for generating very cold plasmas with the both generators: $0.9 \mathrm{MHz}$ and 13.56 $\mathrm{MHz}$. It consists of a copper wire confined in a PVC tube mounted on the male part of the BNC connector. It is covered with a PTFE layer, excepting a length of $1 \mathrm{~mm}$ at its free end. The free end of the plasma electrode is located approximately $1 \mathrm{~mm}$ up-stream of the exit of the PVC tube with an inner diameter of $3 \mathrm{~mm}$. The torch is supplied with plasma gas (helium) at various flow-rates $\left(0-4.51 \mathrm{~min}^{-1}\right)$. The plasma is generated at the free end of the copper wire, which is the single plasma electrode.

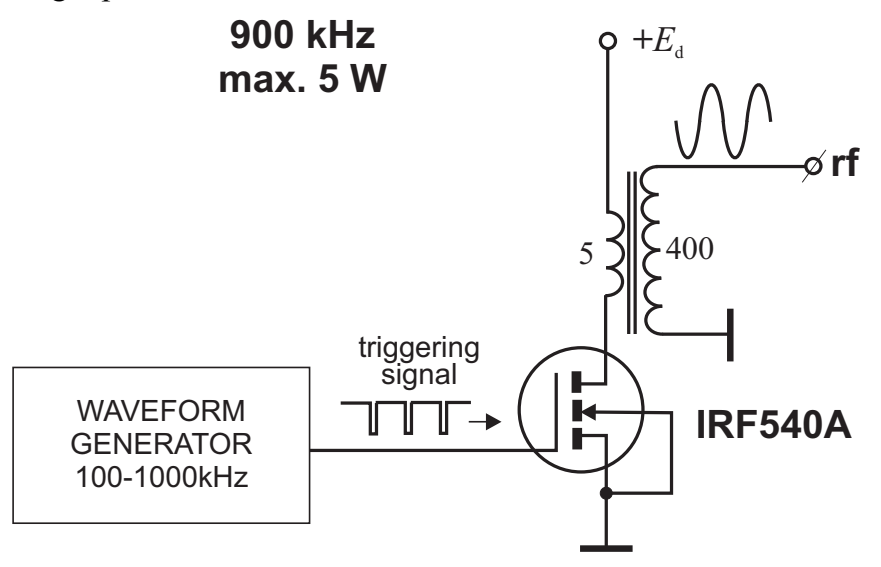

Fig. (13). Generator for very low power CCP.

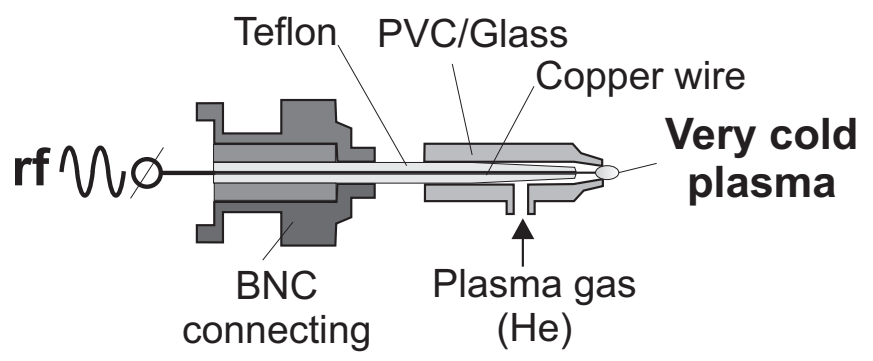

Fig. (14). Torch for very low power CCP.

Recent studies on this kind of plasma [28, 29] shown that at plasma powers lower than $2.5 \mathrm{~W}$, the gas temperature is lower than $75^{\circ} \mathrm{C}$ and the excitation temperature for $\mathrm{He}$ is around $0.2-0.25 \mathrm{eV}$. It was tested as deactivation agent for Escherichia coli cultures and was shown that the plasma is capable of destroying bacterial culture, the overall decimal time (treatment interval necessary to inactivate $90 \%$ of the initial CFUs - colony forming units) is around $25 \mathrm{~s}$. The deactivation capability is better than the other classical sterilization techniques such as dry heat or UV radiation techniques. Other promising applications of this kind of plasma, such as modification of surface properties, are currently under consideration in our laboratory.

\section{CONCLUSION}

It can be concluded that the generation of APPs based on resonant behavior of RLC series circuits represents an interesting alternative in designing and building of classical plasma generators. The APPs generated with our devices can be successfully used in many applications. At this time, the most attractive of them seem to be low power helium CCP, helium (or helium-argon) plasma jet and very low power helium plasma. Further investigations are in progress in our laboratory regarding these plasmas.

\section{ACKNOWLEDGEMENT}

This study was supported by National University Research Council, Romania, Grant IDEI, code 2270.

\section{REFERENCES}

[1] Napartovich AP. Overview of atmospheric pressure sischarges. Producing nonthermal plasma. Plasmas Polym 2001; 6: 1-14. 
[2] Bogaerts A, Neyts E, Gijbels R, van der Mullen J. Gas discharge plasmas and their applications. Spectrochim Acta B 2002; 57: 60958.

[3] Fridman A, Chirokov A, Gutsol A. Non-thermal atmospheric pressure discharges. J Phys D: Appl Phys 2005; 38: R1-R24.

[4] Tendero C, Tixier C, Tristant P, Desmaison J, Leprince P. Atmospheric pressure plasmas: A review. Spectrochim Acta B 2006; 61: 2-30.

[5] Boumans PWGM, De Boer FJ, De Ruiter JW. A Stabilized rf argon-plasma torch for emission spectroscopy. Philips Techn Rev 1973; 33: 50-9.

[6] Anghel SD, Popescu A, Racz F, Tătaru E, Cordos E. Un generator de plasma cuplata inductiv pentru spectroscopia de emisie. Rev Chim 1989; 40: 344-9.

[7] Anghel SD. An rf generator for capacitively coupled plasma at atmospheric pressure. Studia Univ Babes-Bolyai Cluj Physica 1993; XXXVIII: 39-51.

[8] Cordos EA, Anghel SD, Frentiu T, Popescu A. Capacitively coupled plasma with tip-ring electrode geometry for atomic emission spectrometry. Analytical performance and matrix effect of sodium chloride and potassium chloride. J Anal At Spectrom 1994; 9: 63541.

[9] Cordos EA, Frentiu T, Rusu AM, Anghel SD, Fodor A, Ponta M. Analytical characterization of CCP torch with central tube electrode. Talanta 1999; 48: 827-37.

[10] Anghel SD. Physical characteristics and spectroscopical applications of rf CCP in tip-ring geometry at atmospheric pressure. Romanian Reports in Physics 1998; 50: 401-15.

[11] Anghel SD, Darvasi E, Frentiu T, Rusu AM, Simon A, Cordos EA. Characteristic temperatures and electron number densities in an $\mathrm{rf}$ capacitively coupled plasma. Fresenius J Anal Chem 1996; 355 : 250-51.

[12] Frentiu T, Rusu AM, Ponta M, Anghel SD, Cordos EA. Analytical performance of an $\mathrm{rf}$ capaciltively coupled plasma for atomic emission, with tip-ring electrode geometry. Fresenius J Anal Chem 1996; 355: 254-5.

[13] Frentiu T, Anghel SD, Rusu AM, Popescu A, Simon A, Cordos EA. Figures of merit and physical characteristics of a capacitively coupled radiofrequency argon plasma sustained at atmospheric pressure in tip-ring electrode geometry. Acta Chimica Hungarica ACH Models Chem 1999; 136: 131-48.

[14] Frentiu T, Rusu AM, Anghel SD, et al. Radiforequency CCP torch with central tubular electrode and outer ring electrode. Acta Chimica Hungarica - ACH Models Chem 1999; 136: 119-29.

[15] Frentiu T, Anghel SD, Nicola M, Darvasi E, Simon A, Cordos EA. Figures of merit and fundamental processes in analysis of $\mathrm{Ca}$ from liquid samples using an rf CCP torch with tubular and ring electrodes. Croatica Chemica Acta 1999; 72: 763-78.

[16] Frentiu T, Ponta M, Rusu A, Anghel SD, Simon A, Cordos EA. Cadmium determination in sedimented dusty by atomic emission spectrometry with a new radiofrequency capacitively coupled plasma source. Anal Lett 2000; 33(2): 323-35.

[17] Frentiu T, Anghel SD, Simon A, Cordos EA. Study of some matrix effects in low power radiofrequency capacitively coupled plasma with a central tubular molybdenum electrode and a single ring electrode. Acta Chimi Hungarica - ACH Models Chem 2000; 137: 67790.

[18] Frentiu T, Ponta M, Anghel SD, Simon A, Marginean I, Cordos EA. Statistical evaluation of $\mathrm{Cu}, \mathrm{Mn}$ and $\mathrm{Zn}$ determinations in biological samples by radiofrequency capacitively coupled plasma atomic emission spectrometry using the Bland and Altman test. Mikrochim Acta 2003; 143: 245-54.

[19] Frentiu T, Ponta M, Anghel SD, Simon A, Incze AM, Cordos EA. Investigation of medium power radiofrequency capacitively coupled plasma and their application to atomic emission spectrometry for the determination of aluminium in water samples. Mikrochimica Acta 2004; 147: 93-103.

[20] Anghel SD, Frentiu T, Rusu AM, Bese L, Cordos EA. The analysis of conductible solid samples by rf capacitively coupled plasma at atmospheric pressure. Fresenius J Anal Chem 1996; 355: 252-3.

[21] Anghel SD. Generation of a low-power capacitively coupled plasma at atmospheric pressure. IEEE Trans Plasma Sci 2002; 30 : 660-64.

[22] Anghel SD, Simon A, Frentiu T. Spectroscopic investigations on low power atmospheric pressure capacitively coupled helium plasma. Plasma Sourc Sci Technol 2008; 17: 045016.

[23] Anghel SD, Cordos EA, Frentiu T, Popescu A, Simon A. Atmospheric pressure capacitively coupled plasma source for direct analysis of non-conductive solid samples. J Anal At Spectrom 1999; 14: 541-5.

[24] Anghel SD, Simon A, Radu AI, Hidi IJ. Spectroscopic characterisation of a cross-flow plasma jet. Nucl Instrum Meth Phys Res Sect B: Beam Interact Mater Atoms 2009; B 267: 430-433.

[25] Anghel SD, Simon A, Frentiu T. Characterization of a very low Ar CCP. J Anal At Spectrom 2005; 20: 966-73.

[26] Anghel SD, Simon A. An alternative source for generating atmospheric pressure non-thermal plasmas. Plasma Sourc Sci Technol 2007; 16: B1-B4.

[27] Anghel SD, Simon A. Measurement of electrical characteristics of atmospheric pressure non-thermal He plasma. Meas Sci Technol 2007; 18: 2642-8.

[28] Simon A, Anghel SD, Pap J. Optimum working parameters for plasma needle used for bacterial deactivation. J Optoelectron Adv Mater 2008; 10: 2077-81.

[29] Simon A, Anghel SD, Papiu M, Dinu O. Diagnostics and active species formation in an atmospheric pressure helium sterilization plasma source. Nucl Instr and Methods in Phys Research Sect B: Beam Interactions with Materials and Atoms 2009; B 267: 438-41.

This is an open access article licensed under the terms of the Creative Commons Attribution Non-Commercial License (http://creativecommons.org/licenses/by$\mathrm{nc} / 3.0 /$ ) which permits unrestricted, non-commercial use, distribution and reproduction in any medium, provided the work is properly cited. 